\title{
Period Doubling and Spatiotemporal Chaos in Periodically Forced CO Oxidation on Pt(110)
}

\author{
Dagmar Krefting \\ Charité-Universitätsmedizin Berlin, Campus Benjamin Franklin (CBF), Hindenburgdamm 30, 12200 Berlin, Germany \\ Prabha Kaira \\ Fritz-Haber-Institut der Max-Planck-Gesellschaft, Faradayweg 4-6, 14195 Berlin, Germany
}

Harm Hinrich Rotermund

Department of Physics and Atmospheric Science, Dalhousie University, Halifax, NS B3H $3 J 5$ Canada*

(Received 24 March 2008; published 29 April 2009)

\begin{abstract}
Periodic forcing of chemical turbulence in the catalytic $\mathrm{CO}$ oxidation on $\mathrm{Pt}(110)$ can induce a period doubling cascade to chaos. Using a forcing frequency near the second harmonic of the system's natural frequency, and carefully increasing the forcing amplitude, the system successively exhibits spiral wave turbulence, resonant pattern formation, and chaotic oscillations. In the latter case, global coupling induces strong spatial correlation. Experimental results are presented as well as numerical simulations using a realistic model. Good agreement is found between experiment and theory. The results give further insight into the complex nature of reaction-diffusion systems and are of high importance regarding control strategies on such systems. The presented setup enhances the range of achievable dynamical states and allows for new experimental investigations on the dynamics of extended oscillatory systems.
\end{abstract}

Periodic forcing is a common method to synchronize nonlinear oscillators. Entrainment or frequency locking phenomena can be observed in many systems; well-known examples encompass biological, chemical, and physical systems [1-4]. At low forcing amplitudes $a$, entrainment appears, where the forcing frequency $\nu_{f}$ is a rational multiple of the oscillator's natural frequency $\nu_{0}$. The frequency range where entrainment is observed grows with increasing forcing amplitude, as described by the renowned Arnol'd tongues. For single oscillators, the entrainment regimes are regularly shaped at low forcing amplitude $a$, but may get a complex structure at stronger forcing, including period doubling, chaotic regimes, and quasiperiodic behavior [5]. In extended oscillatory systems, the forcing scenario gets even more complex $[6,7]$. For chemical reaction-diffusion systems, where the system can be described as an ensemble of diffusively coupled identical oscillators, parts of the system may be in different dynamical state. This leads to a rich variety of spatiotemporal patterns [8], where the unforced oscillation frequency might be shifted $[9,10]$. A special case is chemical turbulence, diffusion-induced spatiotemporal chaos, where synchronous oscillations get unstable [11]. As a consequence, there exists no longer a well-defined natural frequency $\nu_{0}$, but a certain range of oscillation frequencies is observed. Resonant pattern formation in reaction-diffusion systems have been theoretically investigated covering a defined range of $\nu_{f}$ and $a$ [12-15]. Experimentally, in the lightsensitive Belousov-Zhabotinsky (BZ) reaction, spiral wave oscillations could be entrained $[9,16]$, while in catalytic $\mathrm{CO}$ oxidation on $\operatorname{Pt}(110)$ and electrodissolution of $\mathrm{Ni}$ in sulfuric acid solution periodic forcing was used to control chemical turbulence [17-19]. In both experimental systems, period doubling could be observed within some entrainment regimes [17,20]. Furthermore, numerical simulations of $\mathrm{CO}$ oxidation on $\mathrm{Pt}(110)$ under $2: 1$ and 3:1 forcing, using the realistic Krischer-Eiswirth-Ertl model (KEE), predict period doubling [21,22]. Period doubling cascades to chaos are found numerically in the single oscillator KEE model and in CO oxidation with a porous catalyst [23,24]. But to date, no experiments have given evidence for the observed period doubling being part of a bifurcation cascade to chaotic oscillations, and neither the published numerical simulations of the extended system. In this Letter, we present the complete path of periodically forced CO oxidation on $\operatorname{Pt}(110)$ from chemical turbulence to entrainment, and further to chaotic oscillations via a period doubling cascade. Both experimental and theoretical results are presented for 2:1 resonant forcing, regarding periodicity, pattern formation, and spatial correlation.

Experiments are performed in an ultrahigh vacuum (UHV) chamber with a base pressure of $10^{-10}$ mbar. The setup allows for control of the sample temperature and the partial pressures of the reaction educts, either manually or computer controlled using LABVIEW. Prior to the measurement, the Pt surface is prepared by repeated cycles of argon ion sputtering below $T=470 \mathrm{~K}$, oxygen treatment at $T=$ $570 \mathrm{~K}$, and partial pressure of $p_{0_{2}}=10^{-6} \mathrm{mbar}$, and subsequent annealing up to $T=850 \mathrm{~K}$. For CO oxidation, dosing of the reactants is controlled by an automated gas inlet system. The used reaction parameters are $T=546 \mathrm{~K}$, $p_{\mathrm{O}_{2}}=1.5 \times 10^{-4} \mathrm{mbar}$, and carbon monoxide base pressure $p_{\mathrm{CO}}=6.22 \times 10^{-5} \mathrm{mbar}$. Adsorbate patterns were 
imaged on the sample surface using a photoemission electron microscope (PEEM) [25]. The PEEM yields spatially resolved real-time images of the adsorbate-dependent local work function that can be readily translated into coverage patterns. According to the difference in work function between CO- and O-covered Pt, CO-covered areas appear bright in the PEEM image, whereas O-covered regions are dark. Periodic forcing is applied to the system by a periodic modulation of the carbon monoxide partial pressure $p_{\mathrm{CO}}(t)$

$$
p_{\mathrm{CO}}(t)=p_{0}\left(1+a \sin \nu_{f} t\right) .
$$

The forcing is applied after full development of spiral wave turbulence. The system's temporal response is presented as the mean Fourier spectrum of 512 PEEM images ( $t \approx 25 \mathrm{~s}$ ), obtained at 2500 evenly distributed locations on the observed surface region. The amplitudes of the Fourier coefficients are normalized to the maximum peak. The results for increasing forcing amplitudes $a$ are given in Fig. 1.

Figure 1(a) shows the frequency spectrum of the unforced turbulent system. The natural frequency, defined as the most prominent line, is found to be $\nu_{0}=(0.59 \pm$ $0.03) \mathrm{Hz}$. The forcing frequency is set to $\nu_{f}=1.27 \mathrm{~Hz}$, which is slightly higher than twice the calculated natural frequency. At weak forcing, $a=0.014$, the system oscillates with half of the forcing frequency; the system is frequency locked in 2:1 entrainment [see Fig. 1(b)]. The narrowed frequency peak indicates a decreased degree of disorder compared to the unforced, turbulent state. At stronger forcing $a=0.064$, the system's oscillation is period doubled, indicated by the appearance of the subharmonic line $\nu=\nu_{f} / 4$ and its rational multiples, given in Fig. 1(c). Another increase of the amplitude, $a=0.079$, leads to a further bifurcation within the period doubling cascade. The system shows 8:1 entrainment, locking to a

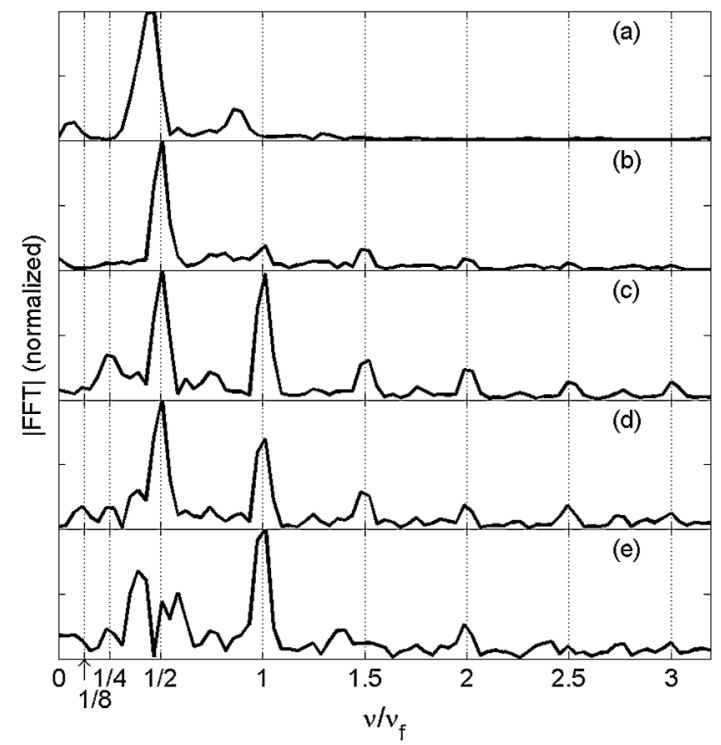

FIG. 1. Mean Fourier spectra at different forcing amplitudes a: (a) 0 , (b) 0.014 , (c) 0.064 , (d) 0.079 , (e) 0.093 . frequency of $v=\nu_{f} / 8$ [see Fig. 1(d)]. Finally, applying a slightly higher forcing amplitude of $a=0.093$, the oscillation is no longer entrained. The Fourier spectrum, given in Fig. 1(e), shows the absence of the subharmonic line at $\nu_{f} / 8$. Lines at $\nu_{f} / 4, \nu_{f} / 2$, and $\nu_{f}$ are still present, but additional frequency components appear in the subharmonic regime. The strong peak between $\nu_{f} / 4$ and $\nu_{f} / 2$ might be related to $3 / 8 \nu_{f}$ within the frequency resolution, but the peak slightly above $\nu_{f} / 2$ (determined to be at $\nu=$ $(0.73 \pm 0.03) \mathrm{Hz})$ cannot be assigned to a rational multiple of the forcing frequency. Therefore, we state a chaotic response of the system at sufficiently high forcing amplitude. Regarding the spatial dynamics, the entrainment of the system is accompanied by suppression of chemical turbulence and cluster formation. At weak forcing, twophase amplitude clusters are formed along with the 2:1 entrainment of the system. The cluster states are preserved during the period doubling cascade and can still be observed in the 8:1 entrained state. In Fig. 2, the spatial amplitude and phase distribution as well as the phase portrait and the phase histogram are shown, obtained for the Fourier coefficients of $\nu_{f} / 8$. Figure 2(a) shows the phase pattern, where mainly two-phase states are observed. They appear in clusters, which are separated by low amplitude boundaries, as can be seen from the amplitude pattern in Fig. 2(b). The phase portrait, given in Fig. 2(c), is mainly line shaped, with two accumulation points with opposite phase. The clustering into two-phase states can clearly be seen in the phase histogram, given in Fig. 2(d).

The conservation of the two-phase cluster pattern is not self-evident. In subharmonic $n: 1$ entrainment, up to $n$ phase states are expected to appear in the extended system. In contrast to a pure homogeneous oscillation, where the diffusion between the surface elements vanish, phase cluster states have strong gradients at the cluster boundaries.

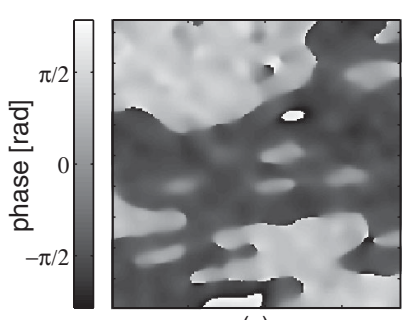

(a)

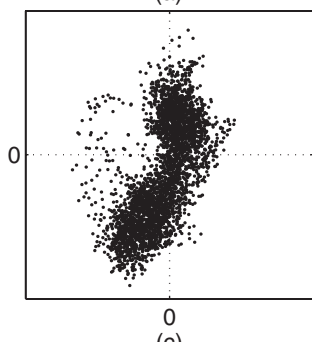

(c)

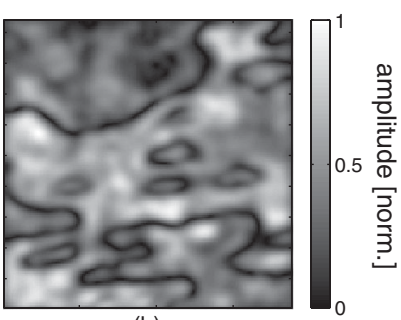

(b)

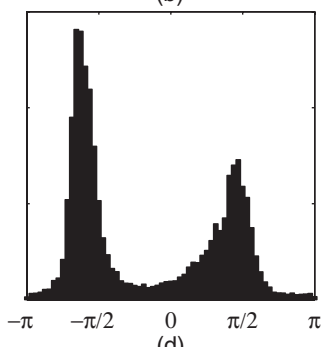

FIG. 2. Two-phase cluster pattern at 8:1 entrainment. (a) Phase pattern, (b) amplitude pattern, (c) phase portrait, (d) phase histogram. 
They may serve as initial locations for the creation of further phase states, for example, obtained numerically for $4: 1$ forcing [26]. Even in the chaotic regime, the cluster pattern is still observable, indicating that the obtained spatial synchronization is not destroyed by the loss of entrainment.

Numerical simulations have been performed for comparison to the experiments. The used KEE model takes adsorption of $\mathrm{CO}$ and oxygen molecules, reaction rates, desorption of $\mathrm{CO}$ molecules, the structural phase transition of the Pt(110) surface, surface diffusion of adsorbed CO molecules, into account. Surface faceting, formation of subsurface oxygen, and the effects of internal gas-phase coupling are not considered. The state of the system is determined by $u(\mathbf{r}), v(\mathbf{r}), w(\mathbf{r})$, describing the surface coverage of $\mathrm{CO}, \mathrm{O}$ and the fraction of the nonreconstructed $1 \times 1$ surface, respectively. The time evolution of these fields are given by

$$
\begin{aligned}
u_{t} & =k_{1} s_{\mathrm{CO}} p_{\mathrm{CO}}\left(1-u^{3}\right)-k_{2} u-k_{3} u v+D \nabla^{2} u \\
v_{t} & =k_{4} p_{\mathrm{O}_{2}}\left[s_{0,1 \times 1} w+s_{0,1 \times 2}(1-w)\right](1-v)^{2}-k_{3} u v \\
w_{t} & =k_{5}\left(\frac{1}{\left.1+\exp \left[u_{0}-u\right) / \delta u\right]}-w\right) .
\end{aligned}
$$

A second-order finite difference scheme is taken for the spatial discretization with a grid resolution of $d x=4 \mu \mathrm{m}$. For integration, an explicit Euler scheme with a fixed time steps $d t=0.001 \mathrm{~s}$ is used. The system size is $400 \times$ $400 \mu \mathrm{m}^{2}$, and no-flux boundary conditions are used. Periodic forcing is applied to the system according to Eq. (1). The initial parameters of the partial pressures are chosen such that the unforced system oscillates and exhibits spontaneously spiral wave turbulence. The model parameters are given in Table I. We have chosen the forcing frequency $\nu_{f}=0.98 \mathrm{~Hz}$ to be near the second harmonic of the most prominent frequency in the extended system in fully developed turbulence $\left(\nu_{0, \text { ext }}=0.51 \mathrm{~Hz}\right)$, rather than twice the single oscillator's natural frequency $\left(\nu_{0, \text { single }}=\right.$ $0.42 \mathrm{~Hz}$ ). The unforced system shows a peak near $\nu=$ $0.51 \mathrm{~Hz}$. Increasing the forcing amplitude, frequency locked 2:1 entrainment is obtained at $a=0.0078$. Period

TABLE I. Model parameters of the KEE model.

\begin{tabular}{lcc}
\hline \hline$k_{1}$ & $3.14 \times 10^{5} \mathrm{~s}^{-1} \mathrm{mbar}^{-1}$ & Impingement rate of $\mathrm{CO}$ \\
\hline$k_{2}$ & $10.23 \mathrm{~s}^{-1}$ & CO desorption rate \\
$k_{3}$ & $283.8 \mathrm{~s}^{-1}$ & Reaction rate \\
$k_{4}$ & $5.86 \times 10^{5} \mathrm{~s}^{-1} \mathrm{mbar}^{-1}$ & Impingement rate of $\mathrm{O}_{2}$ \\
$k_{5}$ & $1.610 \mathrm{~s}^{-1}$ & Phase transition rate \\
$s_{\mathrm{CO}}$ & 1.0 & CO sticking coefficient \\
$s_{\mathrm{O}, 1 \times 1}$ & 0.6 & $\mathrm{O}_{2}$ sticking coeff. $1 \times 1$ phase \\
$s_{\mathrm{O}, 1 \times 2}$ & 0.4 & $\mathrm{O}_{2}$ sticking coeff. $1 \times 2$ phase \\
$u_{0}, \delta u$ & $0.35,0.05$ & Phase transition parameters \\
$D$ & $40 \mu \mathrm{m}^{2} \mathrm{~s}^{-1}$ & CO diffusion coefficient \\
$p_{\mathrm{O} 2}$ & $12.0 \times 10^{-5} \mathrm{mbar}$ & $\mathrm{O}_{2}$ partial pressure \\
$p_{0}$ & $4.6219548 \times 10^{-5} \mathrm{mbar}$ & Base CO partial pressure \\
\hline \hline
\end{tabular}

doubling to $4: 1$ entrainment takes place at $a=0.0102$. A further period doubling to 8:1 entrainment could be found at $a=0.0108$, leading then to chaotic oscillations, similar to the experimental results. The Fourier spectrum gets "noisy," and additional lines appear. At higher forcing near $a=0.06$, the chaotic regime is confined by an inverse period doubling cascade to final 1:1 entrainment. See Ref. [27] for supplementary figures.

Resonant pattern formation is found for all forcing amplitudes. For 2:1 and 4:1 entrainment, two-phase cluster patterns appear, see Fig. 3(b). In contrast to the experiments, we find phase clusters rather than amplitude clusters. Interestingly, we always find a labyrinthine pattern at 8:1 entrainment. An example is given in Fig. 3(c). The transition between two-phase cluster states and the labyrinthine pattern is induced by phase instabilities within the cluster boundary, as mentioned before. However, transition times are longer than $300 \mathrm{~s}$ and might not be fully covered within the experiment. In the chaotic regime, where the oscillation is not entrained to the forcing signal, chemical turbulence continues to be suppressed. Global coupling, induced by the forcing, is assumed to lead to lowdimensional chaos, where the system is spatially correlated. We observe cluster formation similar to 4:1 entrainment [see Fig. 3(b)] although phase fluctuation within the clusters are observed [see Fig. 3(d)]. The spatial correlation $C$ is determined by the cross correlation $k$ of the dynamics at 100 evenly distributed surface locations $x$, averaged over their distance $d$

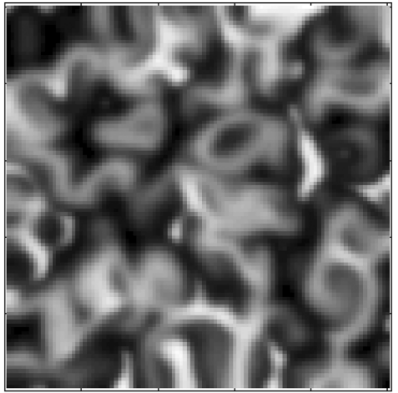

(a)

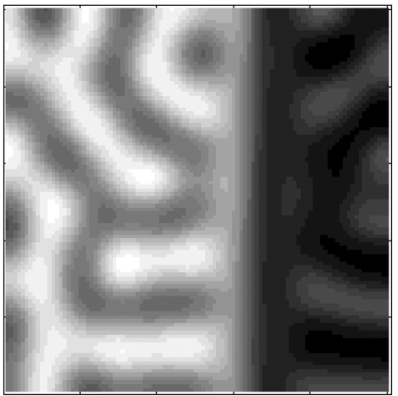

(c)

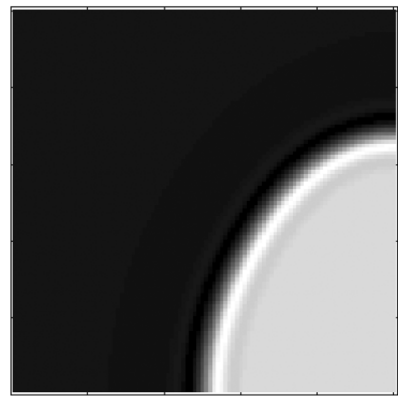

(b)

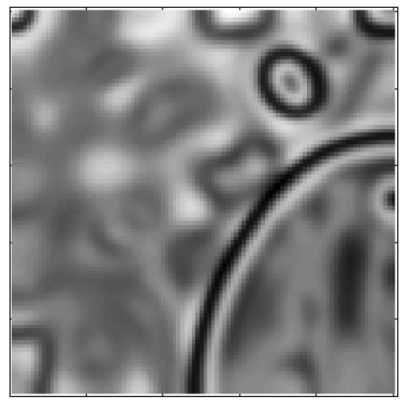

(d)
FIG. 3. Simulated CO coverage for different entrainment states: (a) unforced, turbulent, (b) 4:1 entrainment, (c) 8:1 entrainment, (d) chaotic. Dark gray denotes low, light gray denotes high CO coverage. 


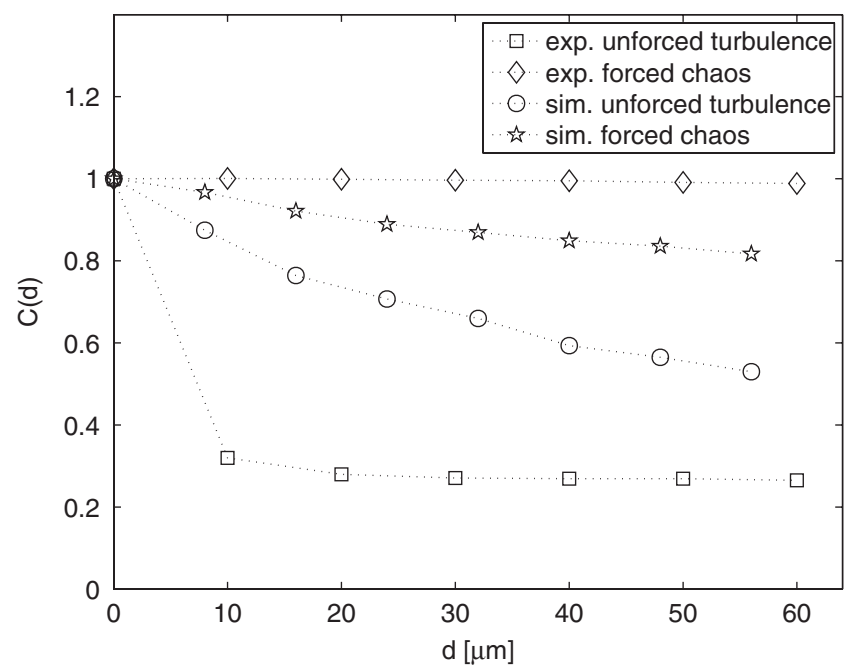

FIG. 4. Mean spatial cross correlation of unforced turbulence and forced chaotic oscillations. Results are given for experimental and numerical data.

$$
\begin{aligned}
C(d) & \left.\left.=\overline{C\left(x_{i}, x_{j}\right)} \quad \forall i, j\left(\left|x_{i}, x_{j}\right| \in\right] d-5, d+5\right]\right), \\
C\left(x_{i}, x_{j}\right) & =\max \left(\left|k\left(x_{i}, x_{j}, \tau\right)\right|\right) / k\left(x_{i}, x_{i}, 0\right), \\
k\left(x_{i}, x_{j}, \tau\right) & =\sum_{t=0}^{N-1} x_{i}(t) x_{j}(t+\tau),
\end{aligned}
$$

where $\tau=N / 2, N / 2-1, \ldots, N / 2$ and $i, j=0,1, \ldots, 99$. The results for unforced and forced spatiotemporal chaos in both the experimental and the simulated system are given in Fig. 4. The cross correlation is normalized to the mean autocorrelation of the sample points. While the cross correlation of the forced experimental system is nearly independent on the distance, it decreases strongly with distance in the unforced turbulent state. The numerical results show the same qualitative behavior, but the difference between the two states is less pronounced. The shorter correlation length in the unforced experiment compared with the simulation can be explained by noise. On the other hand, the higher correlation of the forced experiment might be induced by stronger forcing, as the numerical result is obtained near the lower amplitude boundary of the chaotic regime.

We have demonstrated that attempts to control chemical turbulence by periodic forcing may suppress spatial turbulence, but could lead to chaotic response of the system. The path to chaos is given by a period doubling cascade, which could be experimentally followed by subsequent increase of the forcing amplitude. Numerical simulations support these findings and give further insight into the nature of catalytic $\mathrm{CO}$ oxidation. On one side, the presented results limit the practical use of periodic forcing in order to control chemical turbulence, as chaotic oscillations-even if turbulence is suppressed-is generally not a desired state. On the other hand, once again catalytic $\mathrm{CO}$ oxidation turns out to be one of the most powerful nonlinear systems, where many effects predicted by nonlinear theory can be observed experimentally and reproduced numerically. The system allows for switching between distinct spatiotemporal chaotic states by tuning an easily accessible experimental parameter. Investigation of the complex nature of reaction-diffusion systems - as this work is a part of may lead to improved strategies for control of extended nonlinear systems.

Financial support from the Deutsche Forschungsgemeinschaft through Sonderforschungsbereich 555 Complex Nonlinear Processes and Marie Curie European project Patterns are gratefully acknowledged. We thank Ulrich Parlitz for constitutive discussions.

*Corresponding Author: harm.rotermund@dal.ca

[1] M. Braune and H. Engel, Chem. Phys. Lett. 211, 534 (1993).

[2] A. Karma and V. Zykov, Phys. Rev. Lett. 83, 2453 (1999).

[3] L. Glass, Nature (London) 410, 277 (2001).

[4] S. M. Reppert and D. R. Weaver, Nature (London) 418, 935 (2002).

[5] R. Mettin, U. Parlitz, and W. Lauterborn, Int. J. Bifurcation Chaos Appl. Sci. Eng. 3, 1529 (1993).

[6] J. Kozlowski, U. Parlitz, and W. Lauterborn, Phys. Rev. E 51, 1861 (1995).

[7] H. K. Park, Phys. Rev. Lett. 86, 1130 (2001).

[8] S. Jakubith et al., Phys. Rev. Lett. 65, 3013 (1990).

[9] A. L. Lin et al., Phys. Rev. E 69, 066217 (2004).

[10] P. Kaira et al., Phys. Rev. E 77, 046106 (2008).

[11] Y. Kuramoto, Chemical Oscillations, Waves, and Turbulence (Springer, Berlin, 1984).

[12] P. Coullet and K Emilsson, Physica A (Amsterdam) 188, 190 (1992).

[13] R. M. Mantel and D. Barkley, Phys. Rev. E 54, 4791 (1996).

[14] V. Hakim and A. Karma, Phys. Rev. E 60, 5073 (1999).

[15] V. Petrov, Q. Ouyang, and H. L. Swinney, Nature (London) 388, 655 (1997).

[16] B. Marts et al., Chaos 16, 037113 (2006).

[17] P. S. Bodega et al., New J. Phys. 9, 61 (2007).

[18] I.Z. Kiss, Y. Zhai, and J.L. Hudson, Phys. Rev. E 77, 046204 (2008).

[19] M. Bertram et al., Phys. Rev. E 67, 036208 (2003).

[20] B. Marts et al., Phys. Rev. E 76, 026213 (2007).

[21] K. Krischer, M. Eiswirth, and G. Ertl, J. Chem. Phys. 96, 9161 (1992)

[22] J. Davidsen, A. Mikhailov, and R. Kapral, Phys. Rev. E 72, 046214 (2005).

[23] K. Krischer et al., J. Chem. Phys. 97, 307 (1992).

[24] M. Marek et al., Chaos 16, 037107 (2006).

[25] W. Engel, M.E. Kordesch, H. H. Rotermund, S. Kubala, A. von Oertzen, Ultramicroscopy 36, 148 (1991).

[26] A. L. Lin et al., Phys. Rev. E 62, 3790 (2000).

[27] See EPAPS Document No. E-PRLTAO-102-055917 for supplementary material. For more information on EPAPS, see http://www.aip.org/pubservs/epaps.html. 